\title{
On the flow-phase diagram for discotic liquid crystals in uniaxial extension and compression
}

\section{Gregory Forest ${ }^{*}$ Qi Wang ${ }^{\dagger}$ Hong Zhou}

\author{
October 16, 2000
}

\begin{abstract}
A mesoscopic, extended Doi theory for flows of nematic liquid crystals (LCs) has been successfully applied by Rey to study extensional flow-induced, homogeneous phase transitions both for rod-like [3] and disc-like [4] molecular geometry. Rey analyzed the two order parameters (eigenvalues) of the orientation tensor. Recently the authors [2] generalized the flow-phase diagram (nematic concentration vs. flow rate) for rod-like nematics by analyzing all tensor degrees of freedom, i.e., by coupling the three director (eigenvector) degrees of freedom. Here we record and discuss subtleties of the corresponding diagram for discotic LCs in uniaxial extension and uniaxial compression. We focus here on the induced stable orientation configurations. Uniaxial extension (an idealization of fiber flow) yields a low concentration region of unique oblate uniaxial states at every flow rate; a very small finite region of bi-stable oblate and biaxial states; and the predominant region, encomposing all concentrations above the pure I-N transition and all flow rates, where the only stable steady state is a biaxial pattern. Furthermore, whereas uniaxial states are "unique", all biaxial states occur in a continuous family, corresponding to an arbitrary positioning of the director pair in the plane transverse to the flow axis of symmetry. Uniaxial compression (an idealization of film stretching flow) of discotic LCs exclusively yields stable prolate uniaxial patterns.
\end{abstract}

From Rey [4], the mesoscale orientation of discotic nematic LCs may be described by a rank 2, symmetric, traceless tensor $\mathbf{Q}$. We assume simple extensional flow defined by a velocity field

$$
\mathbf{v}=\nu\left(-\frac{x}{2},-\frac{y}{2}, z\right)
$$

*Department of Mathematics, University of North Carolina, Chapel Hill, NC 27599-3250

†Department of Mathematical Sciences, Indiana University-Purdue University Indianapolis, Indianapolis, IN 46202

$\ddagger$ Department of Mathematics, University of California, Santa Cruz, CA 95064 
where

$$
\nabla \mathbf{v}=\nu \operatorname{diag}\left(-\frac{1}{2},-\frac{1}{2}, 1\right)
$$

For $\nu>0$, the flow stretches along the $z$-axis (uniaxial extension); for $\nu<0$, the flow stretches radially in the entire plane orthogonal to the $z$-axis (uniaxial compression).

The extended Doi theory [1] admits a molecular shape parameter, $\beta$, given by

$$
\beta=\frac{p^{2}-1}{p^{2}+1}
$$

where $p$ is the aspect ratio of an idealized molecular oblate spheroid; $p=1,0, \infty$ correspond to a sphere, flat disc, and cylindrical rod, respectively. For discotic LCs, we fix $0<p<1$, or $-1<\beta<0$.

With the velocity field (1) rigidly enforced, the extended Doi theory for flows of discotic LCs reduces to a flow perturbed tensor ode for $\mathbf{Q}$ in dimensionless form [4]:

$$
\left\{\begin{array}{l}
\frac{d \mathbf{Q}}{d t}=-\mathbf{F}(\mathbf{Q})+\beta \nu \lambda \mathbf{G}(\mathbf{Q} ; \tilde{\mathbf{D}}), \\
\mathbf{F}(\mathbf{Q})=(1-N / 3) \mathbf{Q}-N(\mathbf{Q} \cdot \mathbf{Q})+N(\mathbf{Q}: \mathbf{Q})(\mathbf{Q}+\mathbf{I} / 3), \\
\mathbf{G}(\mathbf{Q} ; \tilde{\mathbf{D}})=\tilde{\mathbf{D}} \mathbf{Q}+\mathbf{Q} \tilde{\mathbf{D}}+\frac{2}{3} \tilde{\mathbf{D}}-2 \tilde{\mathbf{D}}: \mathbf{Q}\left(\mathbf{Q}+\frac{\mathbf{I}}{3}\right),
\end{array}\right.
$$

where the dimensionless velocity gradient $\tilde{\mathbf{D}}$, from $(2)$, is constant,

$$
\tilde{\mathbf{D}}=\operatorname{diag}(-1 / 2,-1 / 2,1), \quad \hat{t}=t / \lambda,
$$

$\lambda$ is the elastic relaxation time of the $\mathrm{LC}$, and $N$ is a dimensionless concentration parameter. The term $\mathbf{F}(\mathbf{Q})$ corresponds to a short-range, Maier-Saupe, excluded-volume interaction, while $\mathbf{G}$ captures the flow-induced nematic response. Since we restrict to homogeneous phase transitions in simple steady flow, the long-range distortional potential is neglected as in $[2,3,4]$.

For rod-like (prolate spheriod) nematics, $0<\beta \leq 1$, the results from [2] apply directly with only a scaling of $\nu \lambda$ by $\beta$. For discotic nematic LCs, $-1<\beta<0$, the flow-phase diagram for flow rate $(\nu)$ vs concentration $(N)$ is achieved by mirror reflection about $\nu=0$ of the diagram in [2]. The analysis in [2] relies heavily on the following tensor basis,

$$
\begin{gathered}
\mathbf{Q}^{(1)}=\left[\begin{array}{ccc}
1 & 0 & 0 \\
0 & 1 & 0 \\
0 & 0 & -2
\end{array}\right], \mathbf{Q}^{(2)}=\left[\begin{array}{ccc}
-1 & 0 & 0 \\
0 & 1 & 0 \\
0 & 0 & 0
\end{array}\right], \mathbf{Q}^{(3)}=\left[\begin{array}{lll}
0 & 1 & 0 \\
1 & 0 & 0 \\
0 & 0 & 0
\end{array}\right], \\
\mathbf{Q}^{(4)}=\left[\begin{array}{lll}
0 & 0 & 1 \\
0 & 0 & 0 \\
1 & 0 & 0
\end{array}\right], \mathbf{Q}^{(5)}=\left[\begin{array}{lll}
0 & 0 & 0 \\
0 & 0 & 1 \\
0 & 1 & 0
\end{array}\right],
\end{gathered}
$$


which provides tensorial eigenfunctions for linearization about all equilibria. From [2, 5] these basis elements correspond to the following homogeneous modes, $\mathbf{Q}^{(1)}$ is a splay mode with respect to the flow axis of symmetry $\mathbf{e}_{z}, \mathbf{Q}^{(2)}$ and $\mathbf{Q}^{(3)}$ are twist modes, $\mathbf{Q}^{(4)}$ and $\mathbf{Q}^{(5)}$ are bend modes. $\mathbf{Q}^{(1)}, \mathbf{Q}^{(2)}$ are equivalent to the order parameter degrees of freedom; $\mathbf{Q}^{(3)}$, $\mathbf{Q}^{(4)}, \mathbf{Q}^{(5)}$ capture the "director" or optical axes degrees of freedom.

Making this correspondence with [2], we deduce the extensional flow-phase diagram for discotic LCs, Figure 1, where $-1<\beta<0$. In Figure 1, the Peclet number Pe $=|\beta| \nu \lambda>0$ corresponds to uniaxial extension and $P e<0$ corresponds to uniaxial compression. Note that the curve $C I$ that separates regimes $V I, V I I$ is not given in [4]. There are many coexisting uniaxial and biaxial steady states in each region; here we report only those that are stable or neutrally stable in Tables 1, 2. Additional unstable states, and their tensor modes of instability, are given in [2].

The characterization of discotic mesophase orientation is given in terms of the eigenvalues $d_{i}$ (order parameters) and eigenvectors $\mathbf{n}_{i}$ (directors) of $\mathbf{Q}$. It is important to remember that the molecular direction $\mathbf{m}$ of a disc-like molecule is the normal to the disc. The eigenvalues of $\mathbf{Q}, d_{i}=\cos ^{2}\left(L\left(\mathbf{n}_{i}, \mathbf{m}\right)\right), 0 \leq d_{i} \leq 1, \sum_{i=1}^{3} d_{i}=1$, provide the birefringence with respect to the optical axes $\mathbf{n}_{i}$, equivalently directors, equivalently eigenvectors, of $\mathbf{Q}$. The spectral decomposition of $\mathbf{Q}$ is

$$
\mathbf{Q}=\sum_{i=1}^{3}\left(d_{i}-\frac{1}{3}\right) \mathbf{n}_{i} \otimes \mathbf{n}_{i} .
$$

The birefringence in the plane of $\mathbf{n}_{i}, \mathbf{n}_{j}$ is $\left|d_{i}-d_{j}\right|$. Isotropic structure corresponds to $d_{1}=d_{2}=d_{3}=1 / 3$; uniaxiality corresponds to one simple eigenvalue $d_{i}$, with $d_{j}=d_{k}$, $i \neq j \neq k$ and isotropy in the plane orthogonal to $\mathbf{n}_{i}$; prolate uniaxiality corresponds to $d_{i}>1 / 3$, so that the molecule normals, $\mathbf{m}$, on average are tilted toward the distinguished optical axis $\mathbf{n}_{i}$; oblate uniaxiality corresponds to $0 \leq d_{i}<1 / 3$. Biaxiality corresponds to $d_{i} \neq 1 / 3, d_{i} \neq d_{j}$, for any $i, j$.

\begin{tabular}{|c||c|}
\hline Region & Type of states \\
\hline \hline VI: & $B$ \\
\hline VII: & $B$ \\
\hline VIII: & $B, O$ \\
\hline IX: & $B$ \\
\hline X: & $B, O$ \\
\hline XI: & $O$ \\
\hline
\end{tabular}

Table 1: A catalog of co-existing stable or neutrally stable steady states of discotic LCs in uniaxial extension. 


\begin{tabular}{|c||c|}
\hline Region & Type of states \\
\hline \hline I: & $P$ \\
\hline II: & $P^{1}, P^{2}$ \\
\hline III: & $P^{1}, P^{2}$ \\
\hline IV: & $P$ \\
\hline V: & $P$ \\
\hline
\end{tabular}

Table 2: A catalog of co-existng stable steady states of discotic LCs in uniaxial compression.

The new information provided here relates to Regions $V I, V I I$ where a prolate uniaxial state in each region suffers two director instabilities (eigenvectors $\mathbf{Q}^{(4)}, \mathbf{Q}^{(5)}$ ) not resolved in [4]. Thus no stable uniaxial states exist in these parameter regimes and Region IX. These unstable modes are the extensional flow analogs of shear-induced tumbling modes.

The main results for discotic LC mesophases can be summarized as follows.

In unaxial extension $(P e>0)$ :

- The only stable uniaxial discotic phases are oblate, denoted by $O$ in Table 1 , in the low-to-moderate concentration regions to the left of the phase transition curve KEDC. This means the plane of discotic molecules on average tilts toward the flow extension axis; the degree of tilt $\left(d_{3}\right)$ is proportional to $P e$, i.e., the flow rate.

- A neutrally stable biaxial state (denoted $B$ in Table 1 ) is the unique orientation structure everywhere to the right of the phase transition curve KEDC, with $P e>0$. The degrees of orientation, i.e. the order parameters, vary continuously as functions of $N$, $P e$. Each pattern $B$ corresponds to a continuous family of patterns, with the directors in the $x-y$ plane not selected from the physics of this model. This translates to one zero linearized eigenvalue of each fixed biaxial state, with linearized tensorial eigenfunction, $\mathbf{Q}^{(3)}$.

- The small region between curves KEDC and EF has bi-stable states, one oblate $(O)$ and one biaxial $(B)$. This is the only parameter region of bi-stability, which is the remnant of the pure nematic bi-stable concentrations $8 / 3<N<3$, segment $F C$ along $P e=0$ in Figure 1.

- The low concentration region $X I$ only has a unique stable oblate uniaxial phase $(O)$, for all flow rates $\nu>0$ and concentrations left of $K E F$ in Figure 1.

- Crossing $E F$ from left to right, a neutrally stable biaxial state appears and the oblate state remains stable. Crossing $F K$ from left to right, the stable oblate state becomes unstable and a neutrally stable biaxial state appears. Crossing $C D E$ from left to 
right, the stable oblate state becomes unstable and the neutrally stable biaxial state remains. Crossing FDJ or $C I$ from left to right, the stable states are unchanged while all states participating in the transition are unstable. In other words, $F D J$ and $C I$ are mathematical bifurcations which are restricted to unstable equilibria. From an experimental point of view, the bifurcation curves are $F E K$ and $C D E K$.

In Figure 2 we depict the geometrical representation of the mesoscopic orientation tensor for fixed flow rate $P e=0.04$ while varying the concentration (equivalently, temperature).

In axial compression $(P e<0)$, relevant for film stretching-type flows of discotic LCs, the only stable patterns are uniaxial prolate phases, denoted by $P$ or $P^{i}$ in Table 2 . All uniaxial states are discrete, with the distinguished director aligned with the flow axis of symmetry. Again, in Figure 1 the experimental boundaries are $A F$ and $A B C$, whereas $F B G$ and $C H$ are "ghost" phase transition curves restricted to unstable equilibria which have no bearing in the experimental bifurcation.

Acknowledgements: The authors are supported by the Air Force Office of Scientific Research, Air Force Materials Command, USAF, under grant numbers F49620-99-1-0003, F49620-99-1-0008. MGF is also supported by the National Science Foundation through grants DMS-9704549 and DMS-0072553. The US Government is authorized to reproduce and distribute reprints for governmental purposes notwithstanding any copyright notation thereon. The views and conclusions contained herein are those of the authors and should not be interpreted as necessarily representing the official policies or endorsements, either expressed or implied, of the Air Force Office of Scientific Research or the US Government. 


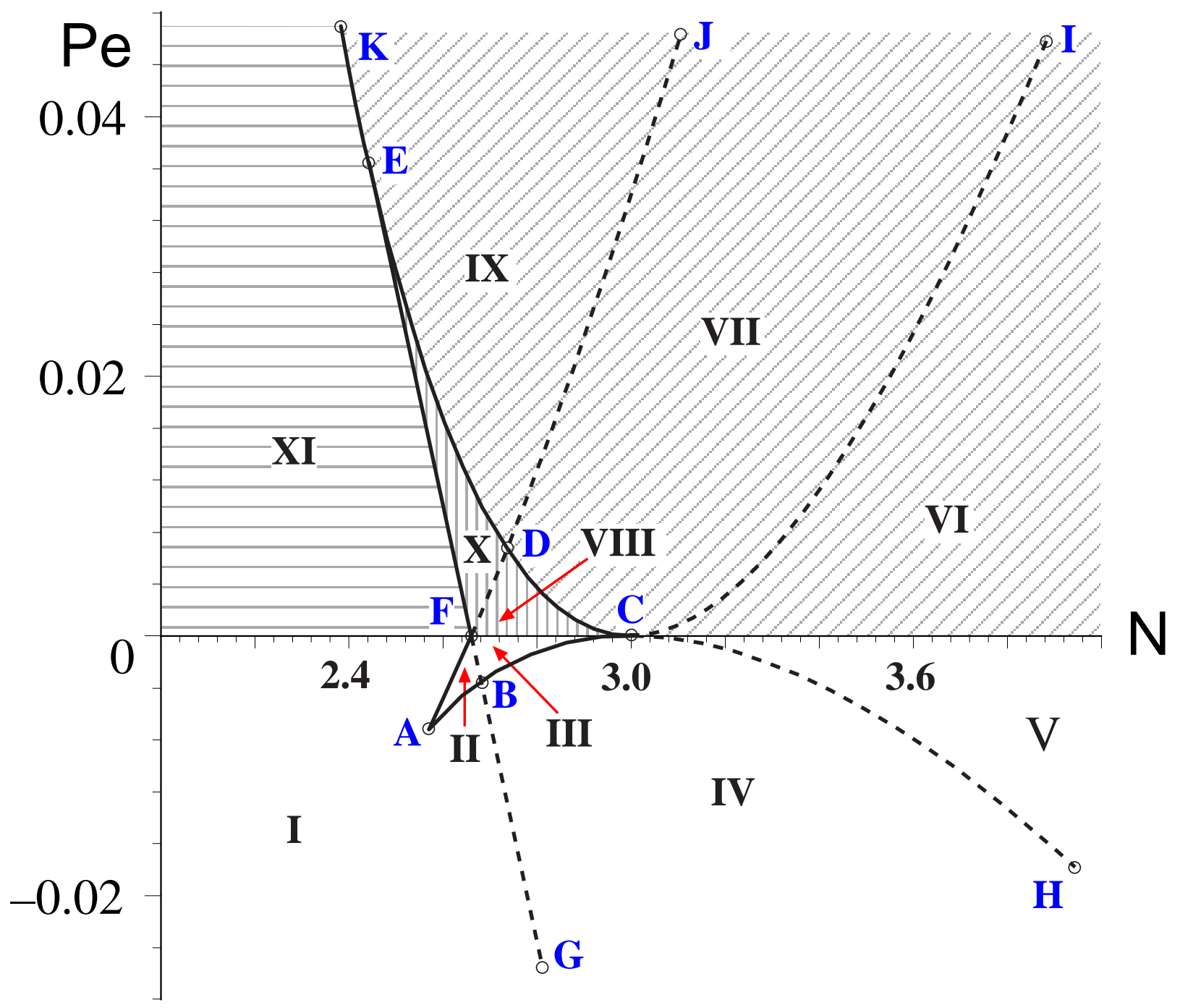

Figure 1: Flow-phase diagram for extensional flow of discotic LCs with molecular shape parameter $-1 \leq \beta<0$, and fixed relaxation time $\lambda$. The vertical axis is the effective Peclet number, $P e=|\beta| \nu \lambda$. $P e>0$ corresponds to uniaxial extension, $P e<0$ to uniaxial compression. 
Region XI

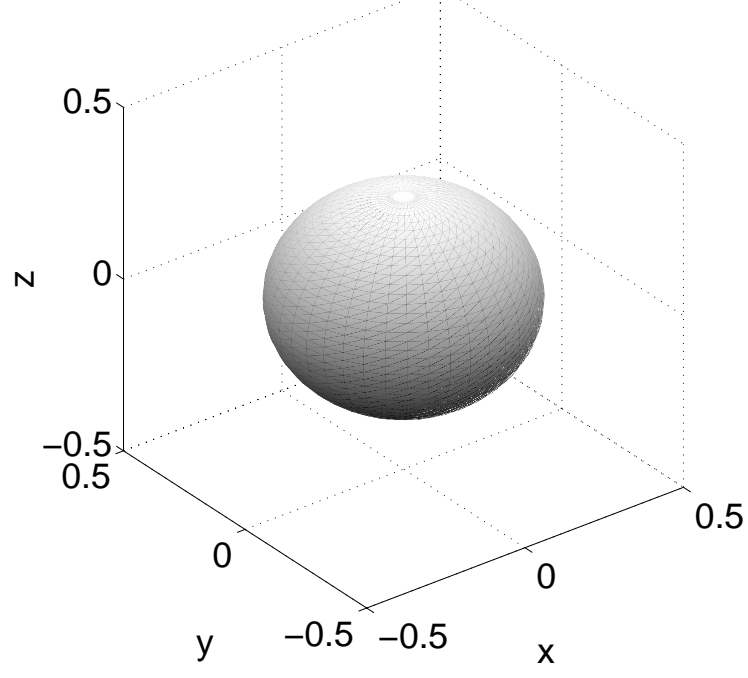

(a) $N=2.2, d_{1}=d_{2}=0.3665$

$$
d_{3}=0.2670
$$

Region VII

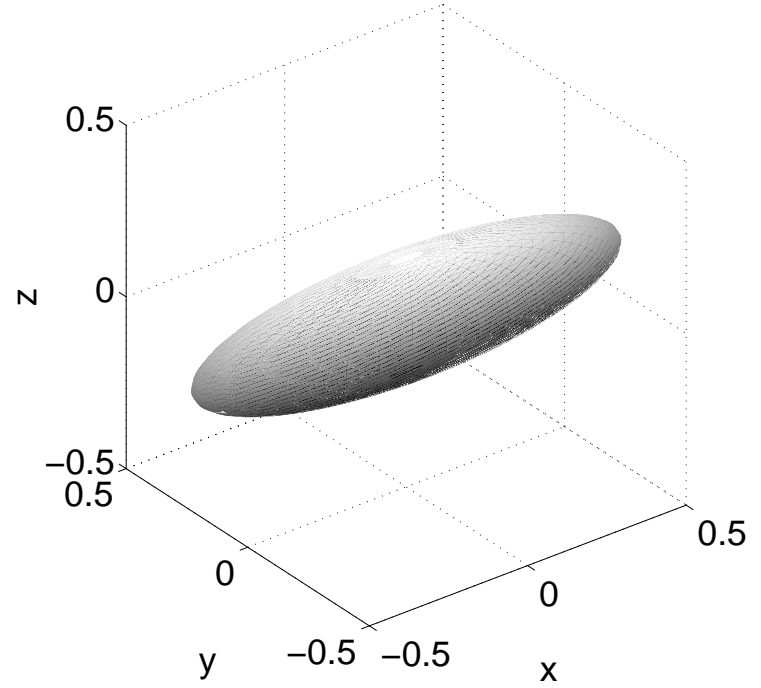

(c) $N=3.3, d_{1}=0.1380, d_{2}=0.7318$

$$
d_{3}=0.1302
$$

Region IX

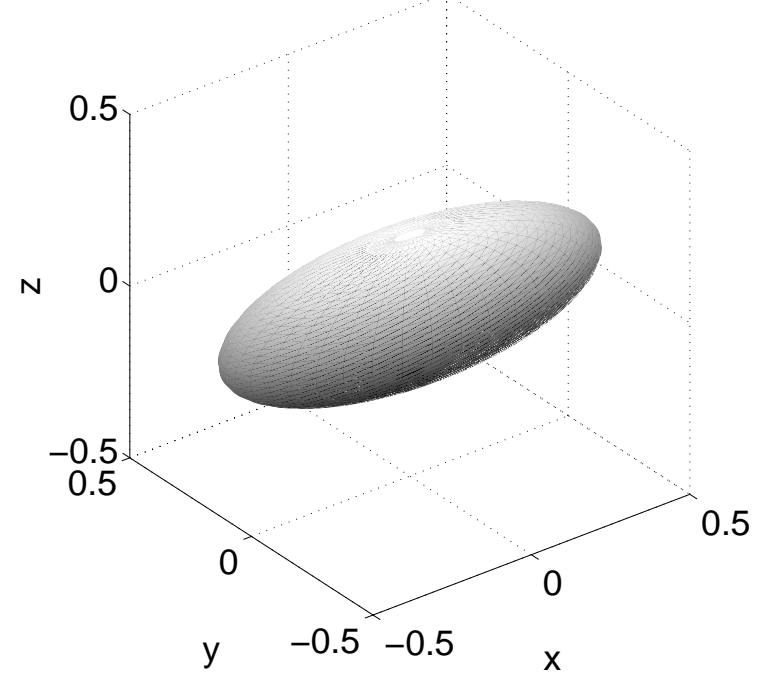

(b) $N=8 / 3, d_{1}=0.2051, d_{2}=0.6095$

$$
d_{3}=0.1854
$$

Region VI

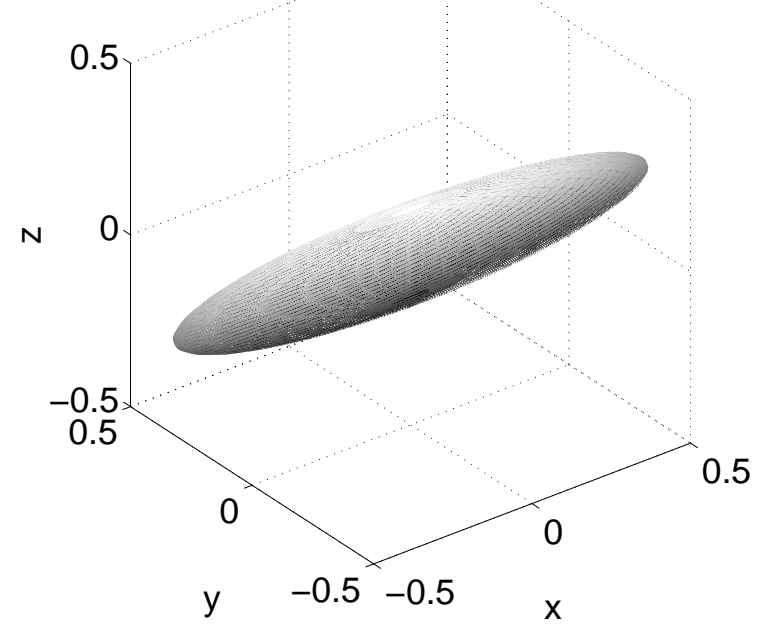

(d) $N=3.9, d_{1}=0.1085, d_{2}=0.7876$

$$
d_{3}=0.1038
$$

Figure 2: Geometrical representation of the mesoscopic orientation tensor in uniaxial extension where $P e=0.04$ is fixed and the concentration $N$ varies. The order parameters determine the degrees of orientation, $d_{i}$, which are the semi-axis lengths. One director is always fixed $\mathbf{n}_{3}=\mathbf{e}_{z}$ parallel to the flow; the remaining directors $\mathbf{n}_{1}, \mathbf{n}_{2}$ are arbitrary, given here as unit coordinate vectors. 


\section{References}

[1] Doi, M. and Edwards, S. F., The Theory of Polymer Dynamics, Oxford University Press (Clarendon), London-New York (1986).

[2] M.G. Forest, Q. Wang and H. Zhou, "Homogeneous biaxial patterns and director instabilities of liquid crystal polymers in axial and planar elongation," Phys. Fluids 12(3), 490-498 (2000).

[3] Rey, A., "Bifurcational analysis of the isotropic-nematic phase transition of rigid rod polymers subjected to biaxial stretching flow," Macromol. Theory Simul. 4(5), 857-872 (1995).

[4] A. Rey, "Bifurcational analysis of the isotropic-discotic nematic phase transition in the presence of extensional flow," Liquid Crystals 19(3), 325-331 (1995).

[5] Shimada, T., M. Doi and K. Okano, "Concentration fluctuation of stiff polymers. III. Spinodal decomposition," J. Chem. Phy. 88(11), 7181-7186 (1988). 